\title{
Indications to tubal reconstructive surgery in the era of IVF
}

\author{
R. Marana • S. Ferrari • A. L. Astorri • L. Muzii
}

Received: 8 August 2007 / Accepted: 9 August 2007 /Published online: 13 September 2007

(C) Springer-Verlag 2007

\begin{abstract}
In recent years, the treatment of tubal infertility has witnessed a shift from reconstructive surgery to in vitro fertilization. However, tubal surgery retains specific advantages, and appropriate preoperative evaluation allows improved selection of patients who are candidates for tubal reconstructive surgery by identifying the patients with good reproductive prognosis. Of pivotal importance in the selection of patients is the intratubal direct evaluation performed at salpingoscopy. Term pregnancy rates of approximately $70 \%$ and $65 \%$ may be obtained in patients with periadnexal adhesions and bilateral distal tubal occlusion, respectively, when a normal tubal mucosa is observed at salpingoscopy.
\end{abstract}

Keywords Tubal infertility · Proximal tubal occlusion · Distal tubal occlusion - Adnexal adhesions .

Tubal reconstructive surgery $\cdot$ IVF

R. Marana $\cdot$ S. Ferrari

Department of Obstetrics and Gynaecology,

Catholic University of the Sacred Heart,

Rome, Italy

\section{R. Marana $(\bowtie) \cdot$ A. L. Astorri}

International Scientific Institute Paolo VI of Research on Human Fertility and Infertility, Catholic University of the Sacred Heart,

Largo Francesco Vito, 1,

00168 Rome, Italy

e-mail: r.marana@tiscali.it

L. Muzii

Campus Biomedico University,

Rome, Italy

\author{
Abbreviations \\ IVF In vitro fertilization-embryo transfer \\ HSG Hysterosalpingography \\ PTO Proximal tubal occlusion \\ DTO Distal tubal occlusion \\ PID Pelvic inflammatory disease
}

\section{Introduction}

Tubal factor infertility accounts for approximately $25-35 \%$ of cases of female infertility [1-3].

Identifiable causes of tubal infertility are postinfectious tubal damage, post surgical adhesion formation, and endometriosis-related adhesions.

The normal process of captation of the oocyte requires a series of prerequisites: the ovarian surface free from adhesions, the fimbrial-ampullary portion of the tube free to embrace the ovary and, beside tubal patency, a normal activity of the ciliated and secretory cells of the tubal mucosa. Furthermore, the muscular layer of the tube must be undamaged and able to contract.

In recent years the treatment of tubal infertility has witnessed a shift from tubal reconstructive surgery to in vitro fertilization-embryo transfer techniques (IVF). Due to the wider availability of assisted reproductive technologies, the number of women with mechanical infertility treated by reconstructive surgery has decreased, most couples being referred to IVF.

Reproductive surgery is performed with the aim of allowing ovum pick-up by restoring normal anatomic relationships between the fimbriae and the ovary. However, even though reproductive surgery may be successful in restoring normal anatomy, it may not be able to restore normal function of the damaged tubal mucosa. 
The percentages of success of the surgical treatment are therefore strictly correlated with the type of preexisting tubal damage, independently of the surgical technique performed.

Recent refinements of laparoscopic instrumentation and increased surgical skills in operative laparoscopy allow laparotomy to be avoided in most instances.

The advent of salpingoscopy, a technique that allows direct visual evaluation of the tubal mucosa, has allowed improved selection of patients who are candidates for tubal reconstructive surgery by identifying the patients with good reproductive prognosis.

The following is an analysis of the various indications to tubal surgery according to the level (proximal or distal) and type of tubal pathology.

\section{Proximal tubal occlusion (PTO)}

Lack of passage of the contrast medium at the level of the intramural-isthmic portion of the fallopian tube during an hysterosalpingogram (HSG) or a laparoscopy with chromopertubation may be due to a true occlusion consequent to postinfectious fibrosis or to an obstruction due to technical artifacts, a spasm of the uterine tubal ostium, a valve mechanism determined by an area of endometrial thickness (focal hyperplasia), or to plugs of amorphous material.

Bilateral PTO is a relatively infrequent finding. We reported [4] that out of 665 patients undergoing laparoscopy with chromopertubation for primary or secondary infertility, only 35 patients $(5 \%)$ had bilateral PTO confirming a previous HSG finding (25 patients bilateral, 10 unilateral with the contralateral tube either distally occluded or absent). Of these patients, 17 refused any further treatment. After a mean follow-up of 25 months, 3 $(18 \%)$ of these patients spontaneously conceived an intrauterine pregnancy; 4 out of 5 patients who underwent a repeated HSG had bilateral tubal patency. Therefore, the diagnosis of bilateral tubal occlusion proved to be incorrect in 7 out of 17 patients $(42 \%)$.

Furthermore, with regard to the etiology of temporary proximal tubal obstruction, a recent paper [5] hypothesizes that small air bubbles, but more likely tubal kinking, may be an explanation of these findings in the patients undergoing HSG in the supine position. In a series of 156 patients, unilateral PTO was diagnosed in $15 \%$ of patients (24 of 156) and bilateral PTO in 3\% (4 of 156). Rotating the patient such that the obstructed tube was inferior to the uterus resolved $63 \%$ of the unilateral PTO, likely by unkinking the tube at the uterotubal junction, thus dramatically lowering the resistance to the flow of contrast medium. The same manoeuvre was less effective in bilateral PTO, where $25 \%$ of the more dependent tubes became patent. Still, this report offers an important contribution to the explanation of "reversible" PTO. The possibility that some PTO are obstructions and not true occlusions is supported by the study of Sulak et al. [6] who in 1987 reported on 18 patients who were found to have bilateral PTO by both HSG and subsequent laparoscopy with chromopertubation and therefore underwent resection of the occluded tubal segment and anastomosis. Resected tubal segments were studied histologically, and in 11 of the 18 cases no tubal occlusion could be demonstrated. In six cases (three with occlusion and three with apparent patency) the tubal lumen contained an amorphous material of unknown etiology, often appearing to form a cast of the tube. The authors were the first to report on such "plugs" and speculated that, if they cause tubal obstruction, this would explain previously published findings of high pregnancy rates in infertility patients after HSG. The suggested mechanism would be, among others, dislodging of tubal mucus plugs.

In 1987 Thurmond et al. [7] described their technique for selective salpingography and fallopian tube recanalization that has since then been widely used to improve diagnosis by injecting contrast medium through a catheter placed in the tubal ostium. This technique allows differentiation of tubal spasm from true occlusion, and can be performed in the same session as the hysterosalpingographic examination that fails to opacify the tubes. In fallopian tube recanalization, a catheter and guide wire system is used to clear proximal tubal obstruction by amorphous debris.

A review [8] evaluating results with this technique in 1,466 patients reports a successful recanalization of the proximal fallopian tube in $71-92 \%$ of recanalization attempted. Pregnancy rates after the procedure have been variable among series, with an average rate of $30 \%$ during follow-up.

In a retrospective study, Al-Jaroudi [9] et al. have recently evaluated the reproductive performance of women after selective tubal catheterization. Ninety-eight infertile women with hysterosalpingographic findings of PTO underwent a repeat hysterosalpingography examination before selective tubal catheterization. Bilateral tubal patency was documented in 14 patients and patency of one of the tubes in 12 others. PTO was confirmed in 72 patients. Successful recanalization of both tubes was achieved in 25 patients $(34.7 \%)$ and successful recanalization of at least one tube was achieved in 44 patients $(61.1 \%)$. Of the 72 patients who underwent selective tubal catheterization, 23 conceived $(31.9 \%)$.

The cumulative probability of conception was $28 \%$, $59 \%$, and $73 \%$ at 12,18 , and 24 months of follow-up, respectively.

The few patients with failure of tubal recanalization may likely have true occlusion caused by fibrotic scarring of the tube from salpingitis, endometriosis, or surgery. Microsur- 
gical resection and tubocornual anastomosis continue to be the standard of care in these cases [10].

In a review of nine case series including 187 patients with PTO, we reported [11] a 49\% term pregnancy rate per patient, with a $4 \%$ risk of ectopic pregnancy after microsurgery by laparotomy.

In 1987, Patton et al. [12] reported on a series of 27 patients with a postpelvic inflammatory disease (PID) bilateral PTO or PTO of the single remaining tube diagnosed both at HSG and laparoscopy with tubal perfusion. Patients were not excluded on the basis of age, extent of tubal disease, duration of infertility, tubal length, or history of prior operation. After an extended follow-up (mean 1,714 days) the possibility of conception was of $46 \%, 65 \%$, and $69.3 \%$ within 1,2 and 3 years from surgery, respectively.

The probability of a conception resulting in a live birth was $27 \%, 47 \%$, and $53.2 \%$ at 1,2 , and 3 years after surgery, respectively. When only patients who did not have a previous surgery for infertility were considered, the conception rate was $75 \%$ with a live birth rate of $58 \%$ after 3 years.

\section{Periadnexal adhesions}

In case of periadnexal adhesions, the classic open-abdomen surgery has been completely replaced by laparoscopic surgery, because it obtains the same results in terms of reproductive outcomes with all the advantages of the laparoscopic approach (better cosmetic result, minor postoperative pain, shorter hospital stay, decreased risk of postoperative infections, quicker return to work).

The intrauterine pregnancy rate reported in the literature after laparoscopic salpingoovariolysis in nonselected patients ranges from $51-62 \%$, and the ectopic pregnancy rate ranges from $5-8 \%[10]$.

Recent prospective studies have demonstrated that the most important prognostic factor in terms of reproductive outcome after reproductive surgery is the status of the tubal mucosa as evaluated by salpingoscopy [13-16].

For salpingoscopy, a $2.8-\mathrm{mm}$ rigid salpingoscope that allows a detailed vision of the tubal ampullary mucosa is used. The salpingoscope is introduced into the abdominal cavity through the operating channel of the laparoscope. The abdominal ostium of the tube is identified and cannulated, and the tubal mucosa is evaluated. At the ampullary level four or five major folds are noted, with minor folds interspersed between them.

The status of the tubal mucosa is classified according to the classification proposed by Brosens et al. [13] as follows: grade I—normal mucosal folds are seen; grade II— the major folds are separated and flattened but otherwise normal (might be considered a grade I distended by increased intraluminal hydrostatic pressure); grade III-focal adhesions are seen between the mucosal folds; grade IV-extensive adhesions are present between the mucosal folds and/or disseminated flat areas are noticed; grade V-there is a complete loss of the mucosal fold pattern.

Grades I and II identify a normal mucosa; grades III to V identify a tubal damaged by a previous pelvic infectious disease.

It has to be stressed that there is no correlation between the score of periadnexal adhesions and intraluminal damage.

Salpingoscopy allows the identification of the patients with normal tubal mucosa who may most benefit from laparoscopic salpingoovariolysis, with a term pregnancy rate of $70 \%$.

Data from Brosens and Marana [14-16] indicate that about $80 \%$ of patients with periadnexal adhesions have a normal tubal mucosa. Therefore, $80 \%$ of the patients with periadnexal adhesions have a normal mucosa, with $70 \%$ chance of a term pregnancy after a laparoscopic salpingoovariolysis. Most of the pregnancies occur within 1 year of surgery.

\section{Distal tubal occlusion (DTO)}

Salpingoneostomy utilizing microsurgical techniques, first described by Swolin [17] in 1967, has been for years the procedure for the treatment of distal tubal occlusion.

In a literature review of 14 series, including 1,275 patients, we reported [18] a cumulative intrauterine pregnancy rate with microsurgical salpingoneostomy by laparotomy of 326/1275 (26\%). The cumulative term pregnancy rate was $239 / 1158(21 \%)$, the cumulative spontaneous abortion rate $54 / 1125(5 \%)$, and the cumulative ectopic pregnancy rate $96 / 1245(8 \%)$.

Ten studies, including 1,128 patients, had complete information on pregnancy outcomes. The cumulative pregnancy rate per patient was $371 / 1128(33 \%)$. Of the pregnancies, $77 \%(284 / 371)$ were intrauterine, $61 \%$ (227/ $371)$ were term pregnancies $15 \%$ (55/371) were spontaneous abortions, and $23 \%$ (87/371) were ectopic pregnancies.

A recent review evaluated five nonrandomized control studies that compared laparoscopic and open microsurgical tubal surgery for treatment of DTO [19]. No significant difference was observed in the intrauterine pregnancy rate between the two groups (laparotomy group: 138/478, 28.9\%; laparoscopy group: 104/336, 30.9\%; combined OR 1.32 [95\% CI 0.58-3.02]).

In three of the studies, sufficient information was given to compare surgical techniques used at different stages of tubal disease. 
Overall, there was no significant difference in the intrauterine pregnancy rate in laparatomy versus laparoscopy in mild tubal disease (laparotomy group: $83 / 253,32.8 \%$; laparoscopy group: 96/243, 39.5\% OR 1.06 [95\% CI 0. 42-2.70]).

For patients with severe stage tubal disease, there was a significantly increased intrauterine pregnancy rate in the laparotomy group $(47 / 210,22.4 \%$ versus $6 / 86,6.98 \%$, OR 2.88 [95\% CI 1.16- 7.16]).

Subsequently, the principles of microsurgery were introduced in the laparoscopic approach for the treatment of distal tubal disease.

Several classifications have been proposed in order to identify the patients that may most benefit from tubal reproductive surgery in DTO. Various parameters are considered, such as the type and extension of periadnexal adhesions, the degree of tubal occlusion, and the status of the tubal mucosa.

In 1988, the American Fertility Society proposed a scoring system in order to allow the comparison of results obtained from different authors. This was based on the following parameters: type and extension of the adhesions and, in addition, for the classification of distal tubal occlusion, thickness and rigidity of the tubal wall, distal ampullary diameter, and the percentage of mucosal folds preserved at the neostomy site. The importance of intraoperative salpingoscopy to visualize the entire length of the ampullary mucosa was recognized. However, salpingoscopic findings were not included in the scoring system as salpingoscopy was being practiced in very few centers.

Numerous prospective studies have recently demonstrated that, also in the case of distal tubal occlusion, the most important prognostic factor is represented by the status of the tubal mucosa. It is therefore important to identify the patients with normal tubal mucosa by means of salpingoscopy.

In fact, prospective studies have demonstrated that patients with normal tubal mucosa (grades I and II) will have a term pregnancy rate of $65 \%$ after salpingoneostomy (compared to $25 \%$ obtained in nonselected patients).

Studies of Brosens and Marana [14-16] report that in cases of distal tubal occlusion, the percentage of patients with normal tubal mucosa range from $35-45 \%$. Therefore, in cases of DTO, 35-45\% of the patients have a normal tubal mucosa, with a $65 \%$ chance of a term pregnancy rate after a laparoscopic salpingoneostomy.

Most of the pregnancies occur in 12-18 months.

In conclusion, based on these findings, in cases of DTO, our current approach would be a diagnostic laparoscopy with salpingoscopy. Laparoscopic salpingoneostomy would then be performed in the patients with normal tubal mucosa.

\section{Reversal of tubal sterilization}

Tubal sterilization is one of the most used contraceptive methods around the world. It has been reported that about $1 \%$ of the patients undergoing this procedure subsequently request a reversal of tubal sterilization.

Tubo-tubal anastomosis is best performed with microsurgical techniques by laparotomy. The precision afforded by this procedure allows precise excision of the occluded segments and exact approximation of each layer of the proximal and distal portions of the tube.

As in the majority of cases the tubal segments are normal, the outcome is an anatomically and physiologically normal tube although slightly shorter.

This leads to a high intrauterine pregnancy rate with a low risk of ectopic pregnancy. Gomel and McComb [10] have reported a cumulative intrauterine pregnancy rate of $70 \%$ in patients who are $<35$ years of age and a $55 \%$ rate in patients who are 35 years of age or more at the time of reversal, with most pregnancies occurring within 18 months after surgery. The ectopic pregnancy rate is approximately $2 \%$.

Recent improvements in laparoscopic microsurgical instrumentation have prompted a few centers to propose tubal anastomosis by laparoscopic access. In a retrospective clinical study, Yoon et al. [20] reported on 202 women who desired reversal of tubal sterilization. In these patients tubal anastomosis was performed by laparoscopy. The cumulative pregnancy rate in the 186 patients for whom follow-up data were available was $60.3 \%, 79.4 \%$, and $83.3 \%$ at 6,12 , and 18 months after surgery, respectively. Five patients (3.2\%) had ectopic pregnancies; one of these patients subsequently conceived an intrauterine pregnancy.

The authors concluded that laparoscopic tubal anastomosis is less invasive and could be an alternative to the procedure by laparotomy.

Gomel and McComb [10] contend that the mechanical disadvantages inherent in laparoscopic surgery will lead inevitably to less precision than that readily attainable by microsurgery by minilaparatomy for any given surgeon. At present there are no randomized trials with sufficient number of patients to answer this question.

\section{IVF results}

According to the American Society for Reproductive Medicine/Society for Assisted Reproductive Technology Registry published in 2007 [21], reporting the results of 79,042 IVF cycles (with and without ICSI) performed in 2001 , the percentage of clinical pregnancy was $32.8 \%$ per initiated cycle, $38.2 \%$ per retrieval, and $40.6 \%$ per transfer. The delivery rates were, respectively, $27.2 \%, 31.6 \%$, and $33.6 \%$. The cancellation rate was $14.1 \%$; the clinical 
pregnancy loss was $17.2 \%$ and the ectopic pregnancy rate $1.8 \%$.

Of the deliveries, $64.1 \%$ were singletons, $32.0 \%$ were twins, $3.7 \%$ were triplets, and $0.1 \%$ were greater than triplet deliveries.

According to the European Society of Human Reproduction and Embriology Registry published in 2007 [22], reporting the results of 365,000 ART cycles performed in 2003, the clinical pregnancy rate per retrieval and per transfer were, respectively, $26.1 \%$ and $29.1 \%$ for IVF, whereas they were $26.5 \%$ and $28.7 \%$, respectively, for ICSI. Incomplete data were available for the analysis per cycle and for term deliveries.

Of the deliveries, $76.7 \%$ were singleton, $22.0 \%$ were twins, and $1.1 \%$ triplets.

The latest results published by the North American and European societies reported here confirm a trend toward better results for assisted reproductive techniques with passing years. The same improvements are not present for the results of tubal surgery. A major improvement with this respect has, however, been made in the field of better patient selection for tubal surgery, following which, for example, as previously discussed, a global 25\% pregnancy rate in nonselected patients with DTO can be brought up to $65 \%$. In the final section of this review, a personal view on the comparison between IVF and tubal surgery is reported.

\section{Discussion}

It is important to underline that while IVF is a 'palliative' technique, which means that it does not eliminate the problem but bypasses it, surgery is curative in the favourable cases with normal tubal mucosa. This allows women to obtain pregnancy naturally, and it is therefore an option for couples with ethical and religious concerns. If successful, surgery allows women to have more than one pregnancy without further treatment, with an abortion rate similar to that of the normal population.

Indications to IVF for 'tubal factor infertility' may not be correct as this diagnosis often proves to be fallacious. In fact, we have demonstrated that the diagnosis of PTO has a high false positive rate due to technical problems, valve mechanism, intraluminal debris, or chronic inflammation. The diagnosis of DTO, although generally accurate, may sometimes be mimicked by ampullary diverticulae, due to a congenital defect of the myosalpinx, that do not need reconstructive surgery and are not incompatible with pregnancy [23].

In a recent study, Hennelly et al. [24] sent a questionnaire to each patient who was known to have delivered an infant after an IVF or ICSI treatment at their universitybased assisted reproduction unit and who had not returned for further therapy. Five hundred fifty questionnaires were sent out. Five hundred thirteen (94\%) responses were received and analyzed. One hundred six (20.7\%) of the 513 respondents reported that they had had a subsequent spontaneous pregnancy. All the pregnancies occurred within 2 years of the IVF/ICSI pregnancy success. The authors underlined that patients entered the program only if they had a valid indication for IVF/ICSI. These patients truly undertook IVF as a last resort. Therefore, it was surprising to find that 19 out of 128 patients with a diagnosis of tubal factory infertility $(14.8 \%)$ later conceived spontaneously.

With respect to financial concerns, it should be considered that, unlike in the USA, in Italy as in other European countries, operative laparoscopy, even for infertility, is fully subsidized by the government health service when performed in a public hospital. On the contrary, IVF is mainly performed in private centres and is not reimbursed either by the government or private insurances.

The risks of tubal surgery are very low and are due to the known complications of anesthesia and surgery. Although low, the risk of complications is present even in IVF, with a reported prevalence of serious cases of ovarian hyperstimulation syndrome of 14 per 1,000 women after the first cycle and 23 per 1,000 after a mean of 3.3 treatments in the 9,175 patients followed by the National Research and Development Centre of Finland [25].

With regard to cumulative pregnancy rate after IVF, in a recent paper Sharma et al. [26] reported a cumulative live birth rate of $66 \%$ following four cycles of IVF. However, the discontinuation rate was very high during the study. Only $36 \%$ of patients continued treatment after the first unsuccessful attempt (dropout rate 74\%); the dropout rate was $61 \%$ after the second attempt, and $69 \%$ after the third attempt.

Lack of success and psychological stress are the main factors in influencing the decision to discontinue treatment with increasing number of attempts [27]. A prospective, cohort study reported that an unexpectedly high percentage of couples who performed IVF discontinued the subsidized treatment before the three cycles that were offered. The majority of these discontinuations were due to psychological stress [28].

Concern has recently been expressed about the health of the children conceived after IVF [29, 30]. It has been reported in singleton ART infants a two-fold increase in risk of perinatal mortality, low birthweight, and preterm birth, about a 50\% increase in small for gestational age, and a 30-35\% increase in birth defects [31]. The same Centre for Child Health Research evaluated all papers published by March 2003 with data relating to the prevalence of birth defects in infants conceived following IVF/ICSI compared with spontaneously conceived infants [32]. Meta-analyses 
of seven reviewer-selected studies and of all 25 studies identified as suitable for inclusion in a meta-analysis suggest a statistically significant $30-40 \%$ increased risk of birth defects associated with ART. The authors conclude that this information should be made available to couples seeking ART treatment.

The guidelines recently approved by the Genetics Committee and the Reproductive Endocrinology and Infertility Committee of the Society of Obstetricians and Gynecologists of Canada for counselling of Canadian women using ART recommend: pregnancy achieved by IVF with or without ICSI are at higher risk for obstetrical and perinatal complications than spontaneous pregnancies; singleton pregnancies achieved by ART are at higher risk than spontaneous pregnancies for adverse perinatal outcomes, including perinatal mortality, preterm delivery and low birth weight; ART has a significant risk of multiple pregnancies; risks of multiple pregnancies include higher rates of perinatal mortality, preterm birth, low birth weight, gestational hypertension, placental abruption, and placenta previa; and that further epidemiologic and basic science research is needed to help determine the etiology and extent of the increased risks of congenital abnormalities associated with ART [33].

An increased risk of congenital malformations in relation to IVF even in singleton infants has been confirmed by a recent review analysing the medical literature update to 2006 [34].

In conclusion, in spite of the recent improvements in the success of IVF, tubal reconstructive surgery remains an important option for many couples. In referral centers, surgery should be the first line approach for a correct diagnosis and treatment of tubal infertility. The success of the surgical treatment depends on careful selection of patients using appropriate diagnostic techniques.

\section{References}

1. Ahmad G, Watson A, Vandekerckhove P, Lipford R (2006) Techniques for pelvic surgery in subfertility. Cochrane Database of Systematic Reviews, April 19; 2: CD 000221

2. The Practice Committee of the American Society for Reproductive Medicine (2006) The role of tubal reconstructive surgery in the era of assisted reproductive technologies. Fertil Steril 86: 31-34

3. Jose-Miller AB, Boyden JW, Frey KA (2007). Infertility. Am Fam Physician 75:849-856

4. Marana R, Muzii L, Paielli FV, Lucci FM, Dell'Acqua S, Mancuso S (1992) Proximal tubal obstruction: are we overdiagnosing and overtreating? Gynecol Endosc 1:99-101

5. Hurd WW, Wyckott ET, Reynolds DB, Amesse LS, Gruber JS, Horowitz GM7 (2003) Patient rotation and resolution of unilateral cornual obstruction during hysterosalpingography. Obstet Gynecol 101:1275-1278
6. Sulak PJ, Letterie GS, Coddington CC, Hayslip CC, Woodward Je, Klein TA (1987) Histology of proximal tubal occlusion. Fertil Steril 48:437-440

7. Thurmond AS, Novy MJ, Uchida BT, Rosch J (1987) Fallopian tube obstruction: selective salpingography and recanalization. Radiology 163:511-514

8. Thurmond AS, Machan LS, Maubon AJ, Rouanet JP, Hovsepian DM, Van Moore A, Zagoria RJ, Dickey KW, Bass JC (2000) A review of selective salpingography and fallopian tube catheterization. Radio Graphics 20:1759-1768

9. Al-Jaroudi D, Herba MJ, Tulandi T (2005) Reproductive performance after selective tubal catheterization. J Minim Invasive Gynecol 12:150-152

10. Gomel V, McComb PF (2006) Microsurgery for tubal infertility. J Reprod Med 51:177-184

11. Marana R, Quagliarello J (1988) Proximal tubal occlusion: microsurgery versus IVF: a review. Int J Fertil 35:338-340

12. Patton PE, Williams TJ, Coulam CB (1987) Microsurgical reconstruction of the proximal oviduct. Fertil Steril 47:35-39

13. Brosens IA, Boeck W, Delattin P (1987) Salpingoscopy: a new preoperative diagnostic tool in tubal infertility. J Obstet Gynecol 94:768-773

14. Brosens IA (1996) The value of salpingoscopy in tubal infertility. J Reprod Med Rev 5:1-9

15. Marana R, Rizzi M, Muzii L, Catalano GF, Caruana P, Mancuso S (1995) Correlation between the American Fertility Society classification of adnexal adhesions and distal tubal occlusion, salpingoscopy, and reproductive outcome in tubal surgery. Fertil Steril 64:924-929

16. Marana R, Catalano GF, Muzii L, Caruana P, Margutti F, Mancuso S (1999) The prognostic role of salpingoscopy in laparoscopic tubal surgery. Human Reproduction 14:2991-2995

17. Swolin K (1967) Fifty fertility operations I. Literature and methods. Acta Obstet Gynecol Scand 46:234-250

18. Marana R, Quagliarello J (1988) Distal tubal occlusion: microsurgery versus in vitro fertilization: a review. Int J Fertil 33:107-115

19. Ahmad G, Watson AJS, Metwally M (2007) Laparoscopy or laparotomy for distal tubal surgery? A meta-analysis. Hum Fertil 10:43-47

20. Yoon TK, Sung HR, Kang HG, Cha SH, Lee CN, Cha KY (1999) Laparoscopic tubal anastomosis: fertility outcome in 202 cases. Fertil Steril 72:1121-1126

21. Society for Assisted Reproductive Technology and the American Society for Reproductive Medicine (2007) Assisted reproductive technology in the United States: 2001 results generated from the American Society for Reproductive Medicine/Society for Assisted Reproductive Technology registry. Fertil Steril 87:1253-1266

22. Nyboe Andersen A, Goossens V, Gianaroli L, Felberbaum R, de Mouzon J, Nygren KG (2007) Assisted reproductive technology in Europe, 2003. Results generated from European registers by ESHRE. Human Reproduction 22:1513-1525

23. Muzii L, Marana R, Mancuso S (1996) Distal fallopian tube occlusion: false diagnosis with hysterosalpingography in cases of tubal diverticulae. Radiology 199:469-471

24. Hennelly B, Harrison RF, Kelly J, Jacob S, Barrett T (2000) Spontaneous conception after a successful attempt at in vitro fertilization/intracytoplasmic sperm injection. Fertil Steril 73:774-778

25. Klemetti R, Sevón T, Gissler M, Hemminki E (2005) Complications of IVF and ovulation induction. Hum Reprod 20:3293-3300

26. Sharma V, Allgar V, Rajkhowa M (2002) Factors influencing the cumulative conception rate and discontinuation of in vitro fertilization treatment for infertility. Fertil Steril 78:40-46

27. Rajkhowa M, McConnell A, Thomas GE (2006) Reasons for discontinuation of IVF treatment: a questionnaire study. Hum Reprod 21:358-363 
28. Olivius C, Friden B, Borg G, Bergh C (2004) Why do couples discontinue in vitro fertilization treatment? A cohort study. Fertil Steril 81:258-261

29. Schieve LA, Rasmussen SA, Buck GM, Schendel DE, Reynolds MA, Wright VC (2004) Are children born after assisted reproductive technology at increased risk for adverse health outcomes? Obstet Gynecol 103:1154-1163

30. Kurinczuk JJ, Hansen M, Bower C (2004) The risk of birth defects in children born after assisted reproductive technologies. Curr Opin Obstet Gynaecol 16:201-209

31. Bower C, Hansen M (2005) Assisted reproductive technologies and birth outcomes: overview of recent systematic reviews. Reprod Fertil Dev 17:329-333
32. Hansen M, Bower C, Milne E, de Klerk N, Kurinczuk JJ (2005) Assisted reproductive technologies and the risk of birth defects - a systematic review. Hum Reprod 20: $328-338$

33. Allen VM, Wilson RD, Cheung A; Genetics Committee of the Society of Obstetricians and Gynaecologists of Canada (SOGC); Reproductive Endocrinology Infertility Committee of the Society of Obstetricians and Gynaecologist of Canada (SOGC) (2006) Pregnancy outcomes after assisted reproductive technology. J Obstet Gynaecol Can 28:220-250

34. Farhi J, Fish B (2007) Risk of major congenital malformations associated with infertility and its treatment by extent of iatrogenic intervention. Pediatr Endocrinol Rev 4:352-357 